\title{
Nonlinear Transition Shift and Write Precompensation in Perpendicular Magnetic Recording
}

\author{
Zheng Wu, H. Neal Bertram, Paul H. Siegel and Jack K. Wolf \\ Center for Magnetic Recording Research, University of California, San Diego \\ 9500 Gilman Drive, MC0401, La Jolla, CA 92093-0401 \\ Email: \{z2wu,nbertram,psiegel,jwolf $\} @$ ucsd.edu
}

\begin{abstract}
The read-write process in perpendicular magnetic recording channels includes a number of nonlinear effects. Nonlinear transition shift (NLTS) arising from previously written transitions is one of these. The signal distortion induced by NLTS is reduced by use of write precompensation during data recording. In this paper, we numerically evaluate the effect of NLTS on the read-back signal by using the model proposed by Bertram and Nakamoto. By means of computer simulation, we examine the effectiveness of two write precompensation schemes in combating NLTS effects in a channel characterized by both transition jitter noise and additive white Gaussian electronics noise. We numerically optimize the precompensation schemes according to channel bit-error-rate, as well as more computationally tractable criteria. Our results suggest that a write-precompensation technique with as few as two adjustable parameters can be very effective against NLTS effects.
\end{abstract}

\section{INTRODUCTION}

In a high density perpendicular recording system, nonlinear effects can distort the read-back signal and degrade the system performance. Nonlinear transition shift (NLTS) induced by demagnetization from previously written transitions is one example. As in longitudinal recording, the NLTS in a perpendicular recording channel can be measured by time or frequency analysis of the read-back signal corresponding to a carefully chosen input data pattern. The distortion caused by NLTS can be reduced by the use of write precompensation, whereby, for specific data patterns, deterministic offsets are added to timing of written transitions. A simple and commonly used precompensation scheme is dibit precompensation, which affects the second transition of a dibit pair. In practice, the timing offsets in write precompensation are optimized empirically in order to minimize the bit-error-rate (BER).

There are very few theoretical results on optimal precompensation of NLTS in recording channels to minimize the BER because of the complex nature of the nonlinear effects. Lim and Kavčić [1] presented a dynamic programming method to optimize write precompensation for a longitudinal recording channel with partial erasure, NLTS and additive white Gaussian noise (AWGN). Their objective was to minimize the mean-squared error (MSE) between the output signal of the noisy, nonlinear channel model and that of the noiseless, linear channel model, rather than to minimize BER. They allowed the use of a different precompensation value for each transition. The optimization procedure and the resulting precompensation scheme would be too complex to implement in a real system, however.

In this paper, we will consider a perpendicular recording channel model including jitter noise, AWGN and NLTS with precompensation. We will use the NLTS calculation model proposed by Bertram and Nakamoto [2], [3]. Two precompensation schemes will be considered: dibit precompensation and a two-level precompensation scheme. We will numerically find the precompensation levels that minimize the BER at the detector output and compare the performance with the case where there is no NLTS in the channel. This result will also be compared to the optimal precompensation levels based upon two other criteria: minimizing the MSE as in [1] and minimizing the variance of the net transition shift.

The paper is organized as follows. Section II gives the channel model. Section III introduces the two precompensation schemes we will study and Section IV gives simulation results and comparisons. Section V concludes the paper.

\section{Channel Model}

We consider a channel model with NLTS, jitter noise and AWGN. Let the channel transition response be

$$
s(t)=V_{\max } \operatorname{erf}\left(\frac{0.954 t}{T_{50}}\right),
$$

where $\operatorname{erf}(\cdot)$ is the error function defined as

$$
\operatorname{erf}(x)=\frac{2}{\sqrt{\pi}} \int_{0}^{x} e^{-t^{2}} d t .
$$

$T_{50}$ is the width at the half maximum of the transition response.

Let $\left\{x_{i}\right\}$ be the input binary data sequence to the channel, $x_{i} \in\{-1,+1\}$. The transition sequence induced is thus $d_{i}=$ $\frac{x_{i}-x_{i-1}}{2}, d_{i} \in\{-1,0,+1\}$. The channel output $z(t)$ can be written as

$$
z(t)=\sum_{i} d_{i} s\left(t+\delta_{i}+a_{i}-i B\right)+n_{W}(t) .
$$


Here, $\delta_{i}$ is the net shift of the transition $d_{i}$ with respect to its nominal location in the recording medium, $a_{i}$ is the random position jitter for transition $d_{i}, B$ is the channel bit spacing (as well as the sampling period), and $n_{W}(t)$ is the electronics noise. For $d_{i}=0$, we set $a_{i}=0$, whereas for $d_{i} \neq 0, a_{i}$ is a zero mean Gaussian random variable with variance $\sigma_{J}^{2}$. The jitter values for recorded transitions are mutually independent. The electronics noise $n_{W}(t)$ is modeled as a zero-mean, AWGN process. The variance of the sampled AWGN $n_{W}(k B)$ is denoted by $\sigma_{W}^{2}$. We define the signal-toAWGN ratio to be $S N R_{W}=10 \log _{10}\left(V_{\max }^{2} / \sigma_{W}^{2}\right)$.

The value $\delta_{i}$ is determined as follows. We can write the net shift as $\delta_{i}=\tau_{i}+\Delta_{i}$, where $\tau_{i}$ is the NLTS induced by previously recorded transitions, and $\Delta_{i}$ is the precompensation value for the transition $d_{i}$. By convention, for $d_{i}=0$, we set $\delta_{i}=\tau_{i}=\Delta_{i}=0$. Fixing the parameters of the medium and head configuration, we can determine $\tau_{i}$ from the locations of the previously written transitions in the medium and $\Delta_{i}$ [2], [3]. Thus, $\tau_{i}$ is a function of the transition sequence $d_{0}, \ldots, d_{i}$, the net shifts of the previously recorded transitions $\delta_{0}, \ldots, \delta_{i-1}$, and $\Delta_{i}$. Given a transition sequence and a precompensation scheme, one can therefore determine the net transition shifts of all the recorded transitions.

Using the Taylor series expansion of the transition response, the channel output can be written as

$$
\begin{aligned}
z(t)= & \sum_{i} d_{i}\left[s(t-i B)+\left(\delta_{i}+a_{i}\right) s^{\prime}(t-i B)\right. \\
& \left.+\frac{\left(\delta_{i}+a_{i}\right)^{2}}{2} s^{\prime \prime}(t-i B)+\cdots\right]+n_{W}(t) .
\end{aligned}
$$

The simulation complexity can be reduced if we approximate the channel output by truncating the Taylor series. We call the result an order-1 approximation when we consider only the first derivative of the transition response:

$$
\begin{aligned}
z(t) \approx & \sum_{i} d_{i} s(t-i B) \\
& +\sum_{i} d_{i}\left(\delta_{i}+a_{i}\right) s^{\prime}(t-i B)+n_{W}(t)
\end{aligned}
$$

and an order-2 approximation when we take the first and second derivatives into account:

$$
\begin{aligned}
z(t) \approx & \sum_{i} d_{i} s(t-i B)+\sum_{i} d_{i}\left(\delta_{i}+a_{i}\right) s^{\prime}(t-i B) \\
& +\sum_{i} d_{i} \frac{\left(\delta_{i}+a_{i}\right)^{2}}{2} s^{\prime \prime}(t-i B)+n_{W}(t) .
\end{aligned}
$$

The accuracy of these approximations will be discussed later.

\section{Precompensation Schemes}

According to the discussion above, for a given transition sequence $\left\{d_{i}\right\}$ one could in principle determine a corresponding set of precompensation values $\left\{\Delta_{i}\right\}$ such that the net shift of all transitions would be zero. However, for a real system, calculation of these precompensation values would be computationally very complex, and the resulting precompensation scheme would be extremely difficult to implement.
TABLE I

NORMALIZED NLTS FOR FOUR TRANSITION PATTERNS

\begin{tabular}{|c|c|c|c|c|}
\hline \hline Transition patterns & $\ldots 001(1)$ & $\ldots 011(1)$ & $\ldots 010(1)$ & $\ldots 000(1)$ \\
\hline NLTS / B & $20 \%$ & $12 \%$ & $8 \%$ & 0 \\
\hline
\end{tabular}

In practice, recording systems usually use only a small number of precompensation values corresponding to selected patterns of recently recorded transitions, which play the most important role in determining the NLTS value of the transition being written. Therefore, these precompensation schemes can not completely eliminate the effects of NLTS. However, as we show in the remainder of the paper, it is possible to find a reasonable tradeoff between complexity and performance by using simple precompensation rules that achieve BER performance close to that of a channel with no NLTS effects. In the following subsection, we introduce two such precompensation schemes.

\section{A. Dibit precompensation}

If we assume that all previous transitions are recorded in their proper positions, the NLTS value of the current transition is determined completely by the preceding bit pattern. The NLTS model [2], [3] indicates that, among all bit patterns, the largest possible NLTS of the current transition is caused by an isolated dibit transition pattern. In NRZI notation, where a transition is represented by a "1" and the absence of a transition by a "0", the dibit transition pattern corresponds to the sequence "...0011" with the current transition being the rightmost.

The simplest precompensation scheme, usually called dibit precompensation, applies a shift only to those transitions with a neighboring transition in the preceding bit position. Using the NRZI description, we can specify the precompensation rule for the $i^{t h}$ transition $d_{i}$ as:

$$
\Delta_{i}= \begin{cases}\Delta & \text { if } d_{i-1} \neq 0 \text { and } d_{i} \neq 0 \\ 0 & \text { otherwise }\end{cases}
$$

The value of $\Delta$ that minimizes the BER can be determined numerically by computer simulation. The results of such an optimization are discussed in Section IV.

\section{B. Two-level precompensation}

Though a transition $d_{i-1}$ has the largest effect on the NLTS $\tau_{i}$ of transition $d_{i}$, the shift $\tau_{i}$ can still depend upon transitions prior to $d_{i-1}$. Table I shows the amount of NLTS, normalized with respect to the bit spacing $B$, for several transition patterns, assuming that previous transitions are recorded in their intended positions. The channel parameters used to calculate these NLTS values are the same as those we will use in the computer simulations of Section IV. The "1" within the parentheses corresponds to the current transition, and all bits preceding those explicitly shown are assumed to be "0". We see that among the four patterns, the most significant transition shift is for the dibit pattern. The next largest NLTS arises when the past two transitions are "11". 
With these NLTS results as motivation, we now propose a two-level precompensation scheme that applies precompensation according to the pattern of transitions in the two preceding bit positions. Specifically, we define the precompensation value as

$$
\Delta_{i}= \begin{cases}\Delta_{H} & \text { if } d_{i} \neq 0, d_{i-1} \neq 0 \text { and } d_{i-2}=0 \\ \Delta_{L} & \text { if } d_{i} \neq 0, d_{i-1} \neq 0 \text { and } d_{i-2} \neq 0 \\ 0 & \text { otherwise }\end{cases}
$$

\section{Simulation Results}

The channel simulations use a pseudorandom input data sequence with a sector size of 5120 bits. Using the NLTS model, we calculate the net transition shifts sequentially. The noisy channel output signal is sampled at multiples of the bit spacing $B$. We use a minimum mean-squared error (MMSE) equalizer design with monic constraint [4]. The equalizer is a 21-tap FIR filter and the equalization target has 3 taps. The equalizer output is passed into a Viterbi detector matched to the target. The BER is measured at the Viterbi detector output.

For the NLTS calculation, we set the medium to softunderlayer spacing to $20 \mathrm{~nm}$, and the medium thickness is set to $10 \mathrm{~nm}$. The channel spacing is $16 \mathrm{~nm}$, corresponding to a linear density of about $1.59 \times 10^{6}$ bits/inch. The remanent magnetization to head field gradient ratio is set to 1.5 . With these parameters, the NLTS of the isolated dibit pattern is about $20 \%$, as indicated in Table I.

\section{A. Dibit precompensation}

According to the NLTS model, the contribution of a previously recorded transition to the shift of a transition currently being written decreases as the distance between the transitions increases. For example, with the system parameters mentioned above, the absolute contribution of a transition $4 B$ away from the current transition decreases to $10 \%$ of that of a neighboring transition that is $1 B$ away. The shift induced by a transition $10 B$ away is only $1 \%$ of that of the neighboring transition, and the relative shift resulting from a transition $20 B$ away drops to $0.1 \%$. Therefore, when calculating the NLTS, we can ignore the influence of the transitions far away from the current transition. We denote by $M$ the number of preceding bits used to calculate the NLTS. The larger $M$ is, the more accurate the calculated NLTS will be. We call $M$ the NLTS window length.

Fig. 1 shows the simulated system BER as a function of the normalized dibit precompensation level $\Delta / B$ for window lengths $M=5,10$, and 20 . The simulation uses the order2 channel output approximation. The channel density $T_{50} / B$ is 1 , the jitter noise $\sigma_{J} / B$ is set to 0.08 , and the signal-toAWGN ratio $S N R_{W}$ is $29 \mathrm{~dB}$. As can be seen, the curves corresponding to the different values of $M$ are almost identical. Therefore, in all of the remaining simulations, we use the NLTS window length $M=10$. Note that the precompensation level that minimizes BER is $\Delta=0.08 B$.

In Fig. 2, we compare the BER results obtained using the exact channel output model with those obtained with the order-1 and order- 2 channel approximations. We see that the BER produced by the order- 1 channel approximation is

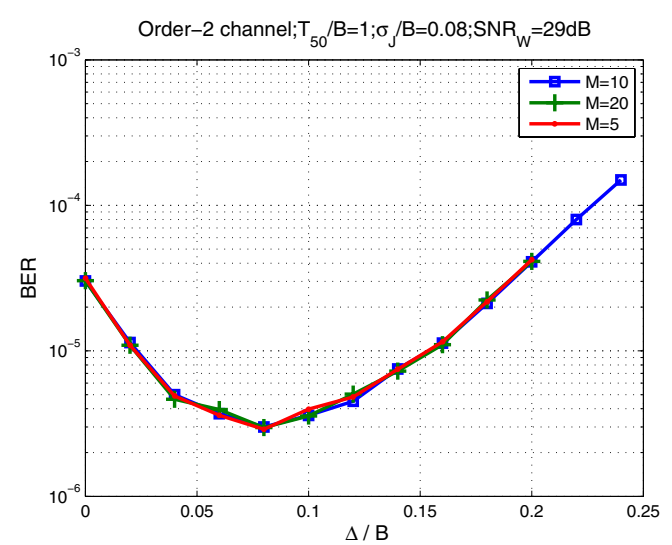

Fig. 1. Influence on BER of NLTS window length $M$.

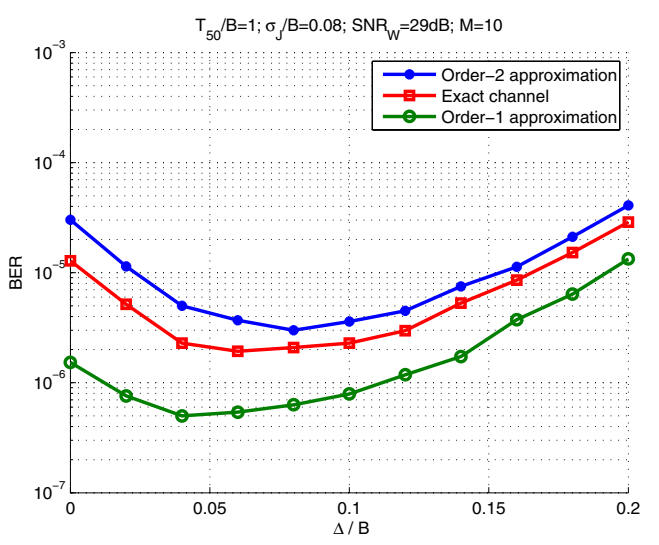

Fig. 2. Comparison of simulated BER for exact channel, order-1 channel approximation, and order- 2 channel approximation.

optimistic relative to that of the exact channel over the entire range of precompensation values, while, in contrast, the BER corresponding to the order-2 approximation is pessimistic over the same range. Since the order-2 estimate is fairly close to the exact channel BER, and is considerably less complex to compute, we will use the order-2 channel approximation in subsequent simulations.

\section{B. Two-level precompensation}

The BER simulation results for the two-level precompensation scheme are shown in Fig. 3. The simulations used the same system parameters as in Fig. 1, with NLTS window length $M=10$. The surface plot shows the BER as a function of the normalized precompensation values $\Delta_{H} / B$ and $\Delta_{L} / B$. The optimal two-level precompensation values are seen to be $\Delta_{H}^{*}=0.09 B$ and $\Delta_{L}^{*}=0.05 B$.

Fig. 4 shows a comparison between the performance of the two-level precompensation scheme and the dibit precompensation. The curve for the dibit precompensation scheme is the same as the order- 2 channel approximation curve shown in Fig. 2. For the two-level precompensation scheme, we fixed $\Delta_{L}$ to be the optimum value $0.05 B$ and plot the BER versus $\Delta_{H} / B$. The point on this curve that achieves the minimum 


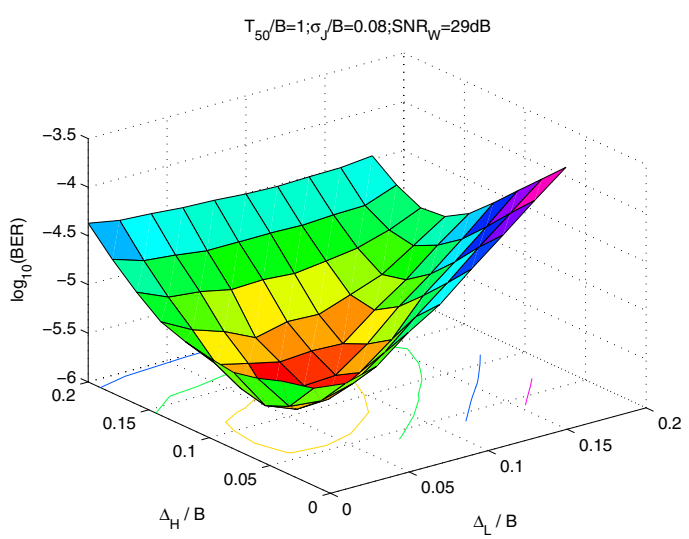

Fig. 3. Surface plot of BER for the two-level precompensation scheme.

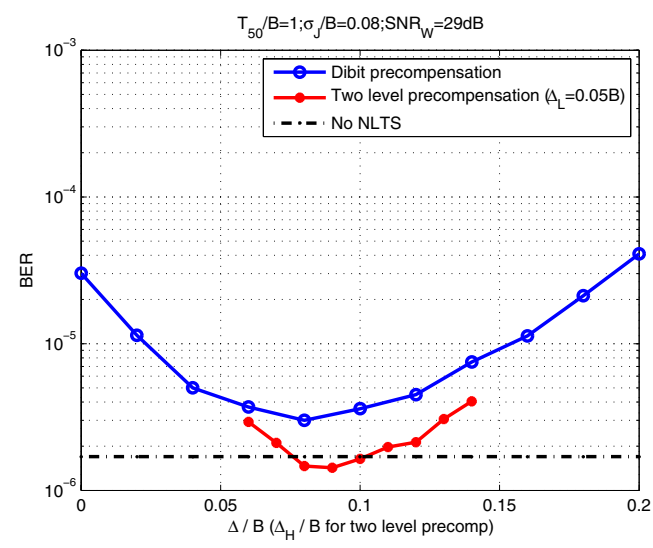

Fig. 4. Comparison of simulated BER for dibit precompensation and twolevel precompensation with $\sigma_{J}=0.08 B$.

BER is thus optimal for the scheme. The horizontal dashdot line represents the BER for a channel with no NLTS and no precompensation but with the same amount of jitter and electronics noise. We see that the dibit precompensation scheme reduces the BER by approximately one order of magnitude compared to a system without any precompensation. There is a small gap between the lowest BER achieved by the dibit precompensation scheme and the BER for the noNLTS case. However, the system with optimized two-level precompensation actually performs better than the system with no NLTS. Specifically, the BERs for the optimal dibit precompensation scheme, the no-NLTS case, and the optimal two-level precompensation scheme are $3.0 \times 10^{-6}, 1.7 \times 10^{-6}$, and $1.5 \times 10^{-6}$, respectively.

In order to verify and better understand these comparative results, particularly the overall superiority of the optimized two-level precompensation scheme, we ran longer channel BER simulations. Indeed, the longer simulations pointed to the same conclusion. In Table II, we list the frequency of occurrence of the dominant error events for the system without NLTS and for the system with NLTS and optimal two-level precompensation. In the simulations, a total of 100, 000 sectors of 5120 bits each were used as channel input. The same
TABLE II

ERROR-EVENT COUNTS FOR SYSTEM WITH NO NLTS AND WITH OPTIMAL TWO-LEVEL PRECOMPENSATION

\begin{tabular}{|c|c|c|}
\hline \hline Error events & No NLTS & Optimal two-level precomp \\
\hline 1 & 414 & 368 \\
\hline-1 & 410 & 385 \\
\hline-11 & 12 & 4 \\
\hline $1-1$ & 4 & 6 \\
\hline $1-11$ & 4 & 1 \\
\hline$-11-1$ & 4 & 2 \\
\hline Total erroneous bits & 888 & 790 \\
\hline BER & $1.73 \times 10^{-6}$ & $1.54 \times 10^{-6}$ \\
\hline
\end{tabular}

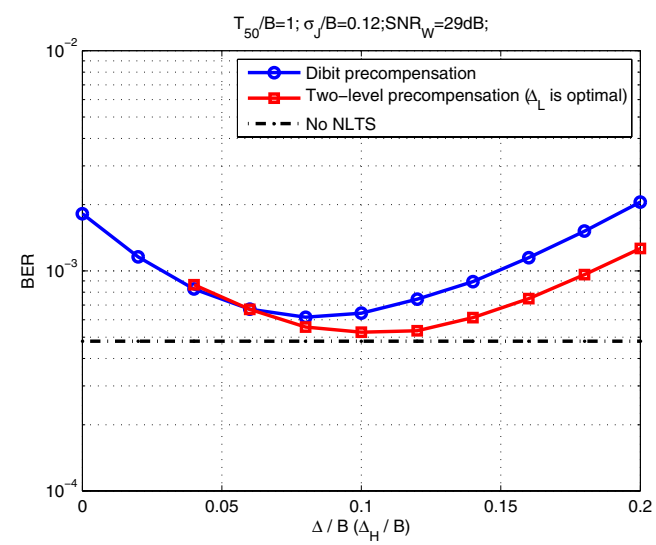

Fig. 5. Comparison of simulated BER for dibit precompensation and twolevel precompensation with $\sigma_{J}=0.12 B$.

pseudorandom input sequence was used in both cases. We also used the same sequence of randomly generated jitter and electronics noise samples. We see from the table that the dominant error event is the single error event for both channels, but the system using two-level precompensation scheme experienced about $9 \%$ fewer of these.

However, this relative performance may not be observed if the channel parameters change. Fig. 5 shows a comparison of BER performance when the precompensation schemes are used on a channel with more severe jitter noise, specifically with $\sigma_{J}=0.12 B$. All the other system parameters remain unchanged. We see that while the optimized two-level precompensation scheme continues to have an advantage over the dibit scheme, it does not outperform the system with no NLTS, although the results are very close.

\section{Other criteria}

In the comparisons above, we used Monte Carlo simulation to find the precompensation values for dibit and two-level precompensation schemes that minimize the BER after the detector. This optimization approach is very time-consuming, however, so it is desirable to consider criteria other than minimum BER in the selection of precompensation parameters. These criteria might not guarantee a minimal BER after detection, but they might produce a suboptimal solution for the precompensation values that nevertheless gives a relatively 
TABLE III

OPTIMAL PRECOMPENSATION VALUES FOR DIFFERENT CRITERIA

\begin{tabular}{|l|c|c|c|}
\multicolumn{4}{|c}{$T_{50} / B=1 ; \sigma_{J} / B=0.08 ; S N R_{W}=29 \mathrm{~dB}$} \\
\hline \hline & BER & Shift Variance & MSE \\
\hline Dibit $(\Delta / B)$ & 0.08 & 0.10 & 0.12 \\
\hline $\begin{array}{l}\text { Two-level } \\
\left(\Delta_{H} / B, \Delta_{L} / B\right)\end{array}$ & $(0.09,0.05)$ & $(0.12,0.08)$ & $(0.14,0.12)$ \\
\hline
\end{tabular}

low BER. We now consider two such alternative optimization criteria.

One of these criteria is commonly known as the meansquared-error (MSE) criterion [1]. The objective is to minimize the MSE between the output signal of the channel with no noise or NLTS and the output signal of the channel impaired by both. The former is given by

$$
y(t)=\sum_{i} d_{i} s(t-i B)
$$

and the latter is given by $z(t)$ as defined in (2), or the approximations in (3) and (4) of Section II. The squared error for each sample is defined by

$$
\epsilon_{k}^{2}=[z(k B)-y(k B)]^{2},
$$

and the MSE is then $E\left\{\epsilon_{k}^{2}\right\}$, where the expectation is taken over all possible samples. It can be approximated by

$$
E\left\{\epsilon_{k}^{2}\right\} \approx \frac{1}{N} \sum_{k=1}^{N} \epsilon_{k}^{2}
$$

With this formulation of the MSE, we can estimate it closely by simulation over a long enough random input sequence.

Another possible optimization criterion is the minimization of the variance of the net transition shifts, $\delta_{i}$. Again, we can accurately estimate this by simulating the effects of NLTS and precompensation over a long random input sequence and computing the sample variance of the observed net transition shifts.

We numerically determined the optimal precompensation values according to these two criteria for the same channel as used to generate the results in Fig. 4. For the dibit precompensation scheme, we found that the value which minimizes the MSE is $0.12 B$, while the value producing the minimum shift variance is $0.10 B$. Note that these precompensation values differ from the one that we previously found to minimize the BER, namely $0.08 B$. In this example, we see that the minimum shift-variance criterion gives an optimal precompensation value that is closer to the value that minimizes the BER. Referring to Fig. 4, we see that, indeed, the BER obtained using this precompensation value is lower than that obtained using the minimum-MSE value.

In Table III, we give the corresponding results for the twolevel precompensation scheme. Referring to Fig. 3, we again find that the precompensation levels that minimize the net shift variance give a lower BER than the levels that minimize the MSE.

It is important to note that the results of such a comparison might not hold for different channel parameters. For example, when we use dibit precompensation, the precompensation value that achieves the minimum BER will be larger when $T_{50}$ is larger, according to our simulations. On the other hand, the variance of net transition shifts does not depend on the value of $T_{50}$. So the precompensation value that minimizes this variance will remain at $0.10 B$. For the minimum MSE criterion, we ran simulations for $T_{50}$ equal to $1.2 B, 1.3 B$, and $1.5 B$. We found that, for all three cases, the optimal precompensation level was $0.12 B$. Now, for the channel with $T_{50}=1.5 B$, we found through Monte Carlo simulation that the precompensation value that minimizes the BER is $0.12 B$. Hence, in this situation, the minimum-MSE criterion is superior to the minimum-shift-variance criterion.

\section{CONCLusions}

We examined through numerical simulation the effects of nonlinear transition shift (NLTS) and write precompensation on the performance of a perpendicular recording channel with random transition jitter and additive white Gaussian electronics noise. Two precompensation schemes were studied: a dibit precompensation scheme and a two-level precompensation scheme. For both, the precompensation levels that minimized BER were obtained by Monte Carlo simulation. Our findings indicate that the two-level precompensation scheme is superior to the dibit precompensation scheme, although the extent of the improvement depends on the channel parameters. Under certain conditions, we found that the optimized two-level precompensation scheme can even provide a BER that is lower than that found in a channel with no NLTS.

We also investigated two other precompensation optimization criteria: minimizing the mean-square-error (MSE) between the channel output signal and the output of an ideal noiseless channel, and minimizing the variance of the net transition shifts. Although these criteria may sometimes yield precompensation values close to those which minimize BER, they have some clear qualitative differences. For example, we found that they each gives values that do not change as the channel parameter $T_{50}$ varies over a fairly wide range, whereas the BER-minimizing precompensation values change substantially.

\section{ACKNOWLEDGEMENT}

The authors would like to thank the Information Storage Industry Consortium (INSIC) and the Center for Magnetic Recording Research at UC San Diego for funding this work.

\section{REFERENCES}

[1] F. Lim and A. Kavčić, "Optimal precompensation for partial erasure and nonlinear transition shift in magnetic recording using dynamic programming," in IEEE Global Telecommunications Conference, Globecom '05, vol. 1, St. Louis, Missouri, USA, Nov. 28th - Dec. 2nd 2005.

[2] H. N. Bertram, Theory of Magnetic Recording. Cambridge University Press, 1994.

[3] K. Nakamoto and H. N. Bertram, "Analytic perpendicular-recording model for transition parameter and NLTS," The Magnetic Society of Japan, vol. 26, no. 2, pp. 79-85, 2002.

[4] J. Moon and W. Zeng, "Equalization for maximum likelihood detectors," IEEE Trans. Magn., vol. 31, no. 2, pp. 1083-1088, Mar. 1995. 